\title{
Los pliegues del liderazgo social y comunitario
}

\author{
Folds of social and community leadership
}

iD Edgar Fernández-Fonseca ${ }^{1}$ \& iDernando Cardona-Sánchez $^{2}$

\begin{abstract}
Resumen
El presente trabajo busca caracterizar las apuestas investigativas que, entre los años 2004 y 2014, se realizaron en torno al liderazgo social y al liderazgo comunitario por parte de los grupos de investigación clasificados en Colciencias, adscritos a universidades y centros de investigación de la ciudad de Bogotá. La lectura crítica y reflexiva del liderazgo social y comunitario, desde el estado del arte, no solo abre el abanico de posibilidades para conocer más sobre lo ya indagado, sino que, además, potencia la formación de líderes que aprenden de líderes, mediante el reconocimiento de las metodologías emprendidas por estos. En los principales acercamientos al corpus documental que se han dado hasta el momento, se encuentra que estos resultan la otra cara de la misma moneda, lo que dificulta su abordaje conceptual y metodológico. Sin embargo, en este sentido, es posible destacar que mientras en el liderazgo social predomina un agenciamiento institucional marcado por una racionalidad técnica y especializada, en el liderazgo comunitario se destacan acciones orientadas al empoderamiento colectivo bajo una racionalidad pragmática que se asume desde el territorio y la identidad.
\end{abstract}

Palabras clave: Liderazgo social; liderazgo comunitario; sociedad; comunidad

\begin{abstract}
The object of this work focuses on characterizing research studies conducted on social leadership and community leadership, between 2004-2014, by research groups classified in Colciencias and attached to universities and research centers in Bogota city. Providing a critical and reflective social and community leadership, from the state of the art, opens up the range of possibilities to get to know what has already been researched, but it also promotes leadership training by means of getting acquainted with the methodologies undertaken by leaders. The main approaches found on to the documentary corpus are the other side of the coin, making difficult the conceptual and methodological approach. However, it can be noted that institutional agency marked by technical and specialized rationality prevails in the social leadership, while on the other hand, in the community leadership, actions directed to collective empowerment under a pragmatic rationality that is territory-and-identity based predominate.
\end{abstract}

Keywords: Social leadership; community leadership; society; community

Tipología: Artículo de investigación científica y tecnológica

Recibido: 04/12/2015

Evaluado: 18/05/2017

Aceptado: 22/05/2017

Disponible en línea: 01/07/2017

Cómo citar este artículo: Fernández-Fonseca, E. \& Cardona-Sánchez, F. (2017). Los pliegues del liderazgo social y comunitario. Jangwa Pana, 16 (2), 197 - 216. DOI: http://dx.doi.org/10.21676/16574923.2133

1. M.Sc. en Investigación Social Interdisciplinaria. Secretaría de Educación Distrital y Corporación Universitaria Minuto de Dios. Colombia. Correo electrónico: efernandezf@uniminuto.edu.co edferfon@gmail.com. ORCID ID: 0000-0002-6685-0441

2. M.Sc. en Educación. Corporación Universitaria Minuto de Dios. Colombia. Correo electrónico: fcardonasan@uniminuto.edu.co fcardonasanchez@ gmail.com. ORCID ID: 0000-0003-0579-0673 


\section{Introducción}

$\mathrm{E}$ presente documento recoge los avances del proyecto de investigación titulado "Estado del arte de las investigaciones sobre liderazgo social y comunitario, realizadas en la ciudad de Bogotá entre los años 2004-2014" realizado por el semillero de investigación Demopraxis, adscrito al Grupo de Investigación Bogotá Sur y al Centro de Educación para el Desarrollo de la Corporación Universitaria Minuto de Dios.

La construcción del estado del arte del liderazgo social y comunitario busca, por una parte, realizar un mapa de la cuestión objeto de estudio con el ánimo de dilucidar las principales perspectivas y corrientes de pensamiento que se destacan en los autores que abordan las investigaciones, así como las metodologías utilizadas al respecto. Y busca, por otra parte, potenciar el desarrollo de habilidades de investigación en los estudiantes miembros del semillero de investigación, dado que el acercamiento a otras trayectorias y recorridos en investigación acercan al estudiante a metodologías y esquemas de análisis e interpretación que sirven de herramientas para futuras investigaciones. Si bien este último factor no cuenta como uno de los objetivos prescritos en la investigación, se constituye como un valor agregado que no se debe desconocer.

En los últimos años, el desborde de literatura sobre el liderazgo social y comunitario ha marcado un campo de estudio en el que están incluidas ramas y disciplinas de las ciencias sociales y humanas que van desde la sociología, la psicología y las ciencias políticas, hasta la administración y la economía. Así también, el número de programas académicos al respecto ha aumentado, sobre todo en áreas de especialización y de diplomados que abordan campos de estudios diversos (inteligencia emocional y liderazgo, gerencia y liderazgo, coaching y liderazgo, gestión comunitaria, innovación y liderazgo, entre otros); lo que da cuenta de un creciente interés tanto de las universidades como de los centros de investigación por el mencionado objeto de estudio. Dichas preocupaciones pertenecen a grupos y redes de investigación que encuentran en sus problematizaciones la posibilidad de ampliar las fronteras del conocimiento sobre el tema. Así, el acercamiento a los mismos brinda la posibilidad de construir un mapa de la cuestión que permite caracterizar, en sus referentes teóricos y metodológicos, los pliegues e irrupciones del liderazgo en los ámbitos de lo social y lo comunitario.

El "liderazgo" y el "líder" como categorías de análisis surgen en la modernidad de la mano de los estudios sobre el sujeto y la constitución de subjetividades, y de su relación con los grupos. En la especie humana existe la tendencia a la formación de colectividades, sin las cuales el individuo, como tal, simplemente no sobreviviría. Como lo mencionan Peter Berger y Thomas Luckmann, "la auto-producción del hombre es siempre, y por necesidad, una empresa social. Los hombres producen juntos un ambiente social con la totalidad de sus formaciones socio-culturales y psicológicas" (2001, p. 71-72). Así mismo, al interior de estas sociedades existen dinámicas de inclusión- exclusión y mandato- subordinación que generan diferencias y desigualdades dentro de los grupos, las cuales, leídas desde las teorías del conflicto social y el funcionalismo de Lewis Coser, permiten la constitución y el fortalecimiento de las identidades, tanto individuales como colectivas. Estas diferencias se encuentran matizadas por la asignación o adjudicación de roles que devienen de las acciones que se realizan al interior del colectivo: "El conflicto es un agente muy importante para establecer la plena identidad y autonomía del ego, o sea, para la diferenciación plena de la personalidad con respecto al mundo exterior (...). El conflicto fija las fronteras entre los grupos internos de un sistema social, robusteciendo la conciencia de grupo y el sentido de la distinción, con lo que se establece la identidad de los grupos dentro del sistema" (Coser, 1961, p. 35-36). Mientras que, por una parte, algunos direccionan estrategias que influyen en la conducta de los otros, por otra parte existen individuos que obedecen los mandatos de determinadas personas. Los primeros reciben 
el estatus de "líder", "dirigente", “jefe", "tutor", etc., siendo sobre la figura de éstos que recae la configuración de un sujeto que tiene la capacidad de movilizar o influir en el comportamiento de los demás miembros del grupo (Lupano, 2003; Zalles, 2010).

El liderazgo, como fenómeno social, se presenta en la interacción de los integrantes de grupos humanos; de ahí que pueda ser concebido como una actividad o un proceso relacional en el que se logra la movilización y gestión de recursos, construcción de estrategias y formas cognitivas, emotivas y valorativas que orientan la conducta de los miembros pertenecientes a los colectivos a través de la emergencia de un sujeto que participa, inspira, dinamiza y problematiza en y para con el grupo (Sánchez, 2002). El liderazgo, entendido como la capacidad de motivar e influir a los demás para llevar a cabo una serie de objetivos, es un potencial que se puede aprovechar, en diferentes ambientes y de maneras distintas, sin recurrir a la figura de autoridad y que se encuentra, por tanto muy ligado al cambio y a la transformación de comunidades y sociedades.

En términos generales, el liderazgo puede ser abordado de diferentes formas: como un rasgo natural en los seres humanos (Platón, Plutarco, Maquiavelo, Antonakis); como una práctica transaccional donde el líder ejerce prácticas de compensación y estimulo con sus subordinados a partir de una lectura de sus necesidades (Palomo, 2010, p. 43), o como un proceso transformacional o adaptativo en el que el grupo logra generar procesos de transformación a partir del trabajo adaptativo que surge del cuestionamiento y la reflexión de la realidad inmediata (Heifetz, 1993). Así también, puede ser abordado a partir de la forma de elección y reconocimiento en el grupo (formal e informal) o a través de las diversas tipologías sobre el líder y su relación con el mismo: ya sea un líder autoritario, que determina las decisiones y da órdenes; un líder democrático, que fomenta la participación y la construcción de iniciativas, es decir un "líder que consulta a sus subordinados para tomar decisiones y acciones en favor de la organización" (Pacsi, Estrada, Pérez \& Cruz, 2014, p. 69); o un líder laissez faire, que de forma pasiva deja que todo pase a partir de las dinámicas del grupo. Este último:

Muestra poca preocupación, tanto por el grupo o por la tarea encomendada, y procura no involucrarse en el trabajo del grupo; constantemente está evadiendo la responsabilidad del resultado obtenido; permanentemente confieren libertad absoluta para que el grupo trabaje según sus criterios y tomen las decisiones correspondientes; este líder proporciona información solo cuando lo solicitan y confiere poder para que el grupo se organice de acuerdo con la iniciativa del grupo (Pacsi et al., 2014, p. 69-70)

Para Muñoz también se encuentra el adhocrático

(...) quien se acopla dependiendo la situación, puede ser quien tome las decisiones o deja decidir al grupo dependiendo la madurez del mismo y características de los subordinados, de su propia capacidad y aptitudes, de las características de la tarea y del tiempo disponible (Muñoz, s.f, p. 2).

Para autores como Sarah Robinson y Ron Kerr, el liderazgo también posee aspectos negativos que afectan el desarrollo de las organizaciones. Desde un punto de vista crítico, realizan aportes teóricos alrededor de los postulados de Pierre Bourdieu, al señalar los efectos negativos que imprimen los líderes carismáticos con el ejercicio de la "violencia simbólica". Así, analizan estudios de casos empíricos donde identifican el potencial coste humano y el posible daño que, a largo plazo, los líderes pueden ejercer a las organizaciones cuando buscan legitimar su poder a partir del carisma, el cual puede ser nocivo o destructivo para el desempeño e identificación de roles en el ámbito organizacional (Robinson \& Kerr, 2009). Bajo este mismo enfoque, se encuentran los aportes de Collado, Jiménez \& EntrenaDurán (2016) quienes señalan que el liderazgo es un fenómeno construido socioculturalmente y que, por tanto, para su estudio deben integrarse las dimensiones subjetivas y objetivas de dicho fenómeno. A partir del constructivismo estructuralista de Bourdieu, en conceptos como 
"capital simbólico", "habitus" y "campus", analizan la forma como los lideres acumulan poder para agenciar acciones que les permiten permanecer o legitimar su capital político.

El liderazgo es un proceso social producto de la sedimentación de las relaciones de poder; de ahí que no se pueda comprender sin adentrarse en una lectura de dichas relaciones, las cuales pueden ir del orden de la subordinación, de la dominación o de la cooperación, con efectos y circunstancias negativas o positivas que inciden tanto en los roles como en las interacciones de los colectivos. Este fuerte contenido político ha sido ampliamente estudiado por el profesor Santiago Delgado, quien destaca que el liderazgo se encuentra matizado por rasgos en el líder como la trayectoria vital, el entorno y la situación para el ejercicio del liderazgo, su agenda política, el papel de los seguidores y la acción política como espacio para la legitimación (Delgado, 2004). Sin lugar a dudas, estos rasgos se constituyen en referentes para analizar las características generales del liderazgo como fenómeno social en el espectro de las relaciones de poder.

En los conceptos de "comunidad" y de "sociedad" se encuentran implícitos las tensiones y debates que existen alrededor del individuo y de lo colectivo, siendo por ende piezas claves para abordar el liderazgo social y comunitario. La búsqueda teórica que permita conocer aspectos sobre la formación o la emergencia del sujeto, que en la dinámica de lo grupal logra direccionar o potenciar procesos de movilización colectiva, lleva a las discusiones que la sociología francesa expresaba en Emile Durkheim con el siguiente interrogante: ¿De qué forma un individuo como unidad biológica se constituye en un ser social? (Durkheim, 1976). Y a partir de éste pensar ¿de qué forma al interior de un grupo florece la figura del líder? De esta forma es posible pensar cómo.

El liderazgo es una actividad social puesto que involucra las relaciones interpersonales que establecen los seres humanos para gestionar y movilizar recursos en pro de apuestas o iniciativas impuestas o compartidas. De ahí la dificultad para demarcar los rasgos que definen al liderazgo social, pues este se entrecruza con el liderazgo ejercido en los ámbitos políticos, económicos y/o administrativos. Un buen elemento para abordar el liderazgo desde lo social es la sociología comprensiva de Weber (2003), quien entiende la sociedad como una red de relaciones sociales en la que interactúan los seres humanos, quienes asumen roles y posiciones y conforman un sistema de símbolos, valores y normas. Sin embargo, la mera suma de los individuos atomizados no conforman "lo social", sino que estos, a su vez, se organizan en grupos o colectivos que los identifican y con los cuales conforman un universo simbólico que los aglutina y les da cohesión (Canto, Castiñeira \& Font, 2009). De ahí que las organizaciones que establecen los individuos se constituyan en el espectro sobre el que lo social toma sentido, es decir que forman micro sociedades que orientan ciertas pautas en el comportamiento de las personas como, por ejemplo, la familia, la escuela, la iglesia, el barrio, etc.

El campo de acción que ayuda a delimitar el liderazgo desde lo social son las organizaciones, dado que en estas se posibilitan procesos orientados al ejercicio colectivo del trabajo adaptativo con o sin autoridad y se establecen relaciones de poder que van desde la subordinación, la dominación o la cooperación. Los estudios organizacionales mencionan que "la organización no solo puede entenderse como una entidad social concreta (ya sea socialmente construida o no), con carácter duradero y con una serie de características y tendencias, sino como un espacio en donde confluyen distintos fenómenos sociales. [Por lo que] teniendo en cuenta esto, la organización es un proceso tedioso de fabricación interminable, más que una cosa sólida y estática" (González, 2014, p.47). De ahí que en las organizaciones se reproduzca el contenido simbólico de la sociedad que las contiene, aunque este no determine sus posibilidades de acción. Por tal motivo resultan tan importantes, pues no solo actúan como eje aglutinador y cohesionador de la sociedad, reproduciendo el orden social, sino que también 
generan acciones que logran dislocar dicho orden y llenar de nuevos contenidos el cúmulo social. Es decir que funcionan como espacios de cohesión y fractura, de reproducción y dislocación, donde los individuos amplían el horizonte de lo interpersonal y construyen su "yo" a partir del "nosotros".

En las organizaciones, los individuos actúan para alcanzar determinados propósitos y asumen roles $\mathrm{y}$ posiciones que determinan sus funciones al interior de las mismas. Dichas posiciones y roles generan tensiones que, en la práctica, manifiestan la carencia o expresión del liderazgo. Es decir, el liderazgo es la forma como son abordados los roles $\mathrm{y}$ las posiciones al interior de las organizaciones.

Se entiende por "liderazgo social" una actividad presente en un determinado tipo de relación grupal organizada en la cual quien direcciona desempeña su rol a partir de una imposición, asignación o elección motivada por sus conocimientos y habilidades en la interacción con los grupos y en el trabajo colectivo.

Por otra parte, el "liderazgo comunitario" es una construcción desde abajo que, como relación social, está cimentada en prácticas que encuentran $\mathrm{su}$ horizonte de acción predominantemente en lo tradicional y en lo emotivo. Sin embargo, esto no constituye que dichas prácticas sean determinantes de la acción, pues si lo fueran se daría lugar a un estancamiento de las prácticas que terminarían por diluirlas en las aguas de lo instrumental. Por el contrario, los sujetos se movilizan porque encuentran un sentido a sus prácticas, las cuales están matizadas por la satisfacción de necesidades colectivas que dan coherencia y aglutinan su quehacer. Tal y como lo enfatiza el profesor Alfonso Torres, al diferenciar lo social de lo comunitario en el "Programa de formadores para la organización comunal" de la Presidencia de la República:

En los lazos comunitarios predomina lo común frente a lo individual y lo íntimo frente a lo impersonal, en las relaciones empresariales prevalece lo individual frente a lo común y el acuerdo racional de intereses por encima de los com- promisos mutuos. Mientras que lo comunitario genera sentido de pertenencia, lo "societal" no (Torres \& Barragan, 2012, p. 19).

Los vínculos con el territorio y los lazos que en él se construyen se convierten en formas de distinguir el liderazgo comunitario. El territorio se convierte no solo en el lugar donde convergen historias de vida compartidas, sino también en el lugar en el que se producen significados sobre los que se articulan formas de asociatividad y cooperación que potencian el mejoramiento colectivo: "concebir el territorio como un espacio de poder, de gestión y de dominio no sólo de individuos, sino de comunidades, entre estas y el Estado, pero también de grupos, organizaciones, empresas locales, nacionales y multinacionales" (Molina \& Ramírez, 2009, p.126). En este sentido, el territorio no es solo un espacio o un lugar, sino un cúmulo de prácticas y relaciones sociales con sentido, donde confluyen y se reafirman identidades colectivas. La vida de las personas que habitan el territorio no ha sido impersonal, ni ha estado atada a un solipsismo instrumental y vacío de cuerpos que habitan casas; por el contrario, sus vidas se encuentran entrecruzadas por lazos de solidaridad y cooperación anclados a la memoria de las mismas luchas y reivindicaciones. Palmo a palmo sus vidas han estado cruzadas por las dinámicas que han logrado tejer entre la casa y el barrio; de ahí que el liderazgo comunitario se constituya en el lugar donde convergen los proyectos personales con los colectivos, pues en este se potencia el encuentro de ideales para un mejor vivir.

\section{Materiales y métodos}

La presente propuesta investigativa, de corte documental, se caracteriza por trabajar metodológicamente desde el estado del arte. Para su desarrollo, se organizó en las siguientes fases, a saber: fase preparatoria, fase descriptiva, fase interpretativa y fase de construcción de sentido teórico global. En la primera, se reconocieron los elementos teóricos que sustentan el proceso investigativo del estado del arte, con el cual se 
identificó y contextualizó el objeto de estudio. Así también, se diseñaron las fichas de trabajo y se entrenó a los investigadores participantes en su uso. Se construyó el corpus documental, el cual fue tomado de la identificación de los grupos de investigación clasificados en Colciencias que realizaron investigaciones sobre liderazgo social y comunitario y las publicaron en revistas, capítulos de libro y libros.

Durante la etapa de identificación de los grupos de investigación se discriminaron aquellos adscritos a diferentes universidades de Bogotá y que pertenecían a escuelas o facultades de Ciencias Sociales y Humanas, particularmente de carreras como: Sociología, Trabajo Social, Psicología, Administración de Empresas y Economía. Una vez identificados, se ingresó a la base de datos de Colciencias para identificar a quienes han realizado investigaciones sobre liderazgo. Para este propósito, se revisaron los libros, capítulos de libro y artículos de revistas científicas que fueron publicados en los años de referencia, 2004- 2014.

De igual modo, los resultados de la indagación preliminar permitieron evidenciar un total de 27 grupos de investigación identificados. De estos, 7 se encuentran en la categoría A, 5 en la categoría B, 9 en la categoría C, 4 en la categoría D y 2 son reconocidos. Estos grupos, con un total de132 artículos publicados en revistas, 37 capítulos de libro y 36 libros resultados de investigación (Tabla 1).

En la segunda fase, se tuvo como objeto extractar los datos pertinentes de las unidades de análisis y someterlas al proceso de revisión, reseña y descripción. Para ello, se utilizaron fichas de registro mediante la elaboración de resúmenes analíticos especializados en los que se buscaba referenciar qué describían los textos consultados acerca del liderazgo social y comunitario, la metodología utilizada, y qué idea se tenía en ellos sobre el líder como sujeto. En la tercera fase, se elaboraron las categorías de análisis y se realizó el planteamiento de la hipótesis o de las afirmaciones útiles para la construcción teórica a partir de tópicos identificados al momento de organizar la información según el año de producción, el tipo de documento y la clasificación del grupo en Colciencias. Esto permitió hallar algunas similitudes o tópicos que identificaban y diferenciaban los documentos para que, finalmente, se pudiera realizar una revisión de conjunto con el fin de formalizar el estado actual del conocimiento de manera global y, de esta forma, orientar nuevas perspectivas de investigación.

Para el análisis se identificaron cuatro espacios o campos de interpretación sobre el liderazgo social y comunitario, los cuales forman pares de comprensión. Por un lado, el poder y la motivación $\mathrm{y}$, por otro lado, el sujeto y lo colectivo.

Lamayoría de las universidades a las que pertenecen estos grupos son: Pontificia Universidad Javeriana, Universidad Pedagógica Nacional, Universidad del Externado, Corporación Universitaria Minuto de Dios, Universidad de la Salle, Universidad Militar Nueva Granada, Universidad Nacional de Colombia, Fundación Universitaria Monserrate, Universidad Piloto, entre otras.

Tabla 1. Identificación de grupos de investigación, publicaciones e instituciones que los avalan.

\begin{tabular}{|c|c|c|}
\hline \multicolumn{2}{|c|}{ Relación Grupos, publicaciones e Instituciones } \\
\hline $\begin{array}{c}\text { Total } \\
\begin{array}{c}\text { Universidades o } \\
\text { instituciones que } \\
\text { avalan }\end{array}\end{array}$ & $\begin{array}{c}\text { Total } \\
\text { Grupos de } \\
\text { Investigación }\end{array}$ & $\begin{array}{c}\text { Total } \\
\text { Publicaciones }\end{array}$ \\
\hline 19 & 27 & 205 \\
\hline
\end{tabular}

Fuente: Elaboración propia de los autores

\section{Resultados y Discusión}

El acercamiento al liderazgo social y comunitario, a través del estado del arte, permite la elaboración de un mapa de la cuestión sobre el que se describen las principales corrientes y enfoques de pensamiento sobre el tema y, a la vez, se identifican 
las principales metodologías y perspectivas analíticas que la comunidad académica utiliza en sus prácticas investigativas. Este ejercicio, por un lado, potencia el desarrollo de habilidades de investigación y, por el otro, abre el caleidoscopio de las experiencias con el fin de construir nuevas problematizaciones sobre el objeto de estudio. Comprender las discusiones y caracterizaciones que se han realizado sobre el liderazgo social y comunitario, desde la postura asumida en el presente trabajo, acerca a futuros investigadores al objeto de estudio desde una perspectiva interdisciplinar. De ahí que la revisión de lo ya indagado brinde herramientas para posteriores pesquisas. Así lo afirma la profesora Olga Lucía Vélez al referirse a los estudios documentales:

(...) parten del conocimiento acumulado, lo organizan, lo contrastan con las distintas tendencias del área objeto de investigación y con las comunidades vinculadas a él para encontrar nuevas comprensiones; facilitan el acceso y el avance del conocimiento; contribuyen a la consolidación de comunidades académicas y a la constitución de redes[...] finalmente se infiere como resultado de esta actitud investigativa permanente, que los estados del arte son fuente de aprendizaje permanente (Vélez, 2002, p.194).

Resulta complejo y obtuso abordar el liderazgo en todas sus acepciones, pues su campo de acción hace parte de los meandros de lo humano. Sus ámbitos desbordan el trabajo que aquí se presenta, debido a que desde ramas del conocimiento y disciplinas como la economía, la administración, las ciencias militares, la psicología, el trabajo social y la ciencia política se aborda dicho fenómeno encontrando que cada una, desde sus abordajes teóricos y metodológicos, llena de sentidos propios las dinámicas y problematizaciones con las que se construye dicho objeto de estudio. Es decir que cada disciplina ofrece una perspectiva que enriquece y amplía la frontera del conocimiento sobre el liderazgo, por lo que se ha llegado, incluso, a la formación de unidades de análisis multidisciplinares que han ampliado la vertiente de estudios sobre el liderazgo.
La multiplicidad de miradas y la concepción en torno al liderazgo comunitario y social no impiden que se pueda presentar un mapa sobre el estado actual de la cuestión, particularmente en lo qué ha sido catalogado, a veces sin distinción entre uno y otro, como liderazgo social y liderazgo comunitario. Esta mirada requiere ciertas aclaraciones: en primer lugar, el liderazgo como fenómeno social no debe ser naturalizado al punto de que el concepto sea objeto de la falacia de la cosificación; esto es, el considerar que entidades abstractas puedan ser convertidas en identidades lógicas adscritas a un marco teórico o conceptual. De ahí que este sea asumido como una "relación", lo cual implica que solo es posible en el horizonte de las interacciones de los seres humanos, quienes con sus prácticas sociales llenan de sentido los significados que orientan dichas prácticas $\mathrm{y}$, por ende, logran comprenderlas como parte del entramado social en constante transformación. En segundo lugar, el liderazgo social y el liderazgo comunitario son dos caras de la misma moneda; de ahí que no exista una postura que los separe y los presente como independientes uno del otro: ambos hacen parte de la compleja red de relaciones y manifestaciones con las que se comprende el liderazgo como fenómeno humano.

Como ser social, el ser humano necesita de los otros para desarrollar todas sus capacidades y habilidades sociales (comunicación, empatía, colaboración, etc.), sin las cuales su interacción social se vería limitada, pues se construye diariamente en la tensión entre lo individual y lo colectivo, entre aquello que desea ser y eso que lo vincula al mundo social, a un espacio en particular. Nadie escapa a dicha tensión, pues la vida oscila entre una y otra orilla. La fuerza del grupo por diluir al sujeto construye un horizonte de significados y valores con los que se crea una visión y orden social del mundo al punto de que, en ocasiones, llegan a naturalizarlo como algo dado e inmodificable. Por su parte, el sujeto recibe este ordenamiento del mundo, el cual no ocurre de forma hermética sino que, por el contrario, logra crear dislocaciones o pliegues subjetivos 
con los que consigue romper los sentidos que han sido naturalizados y llenar de nuevos significados el entramado social. Se trata de una espiral en la que la reproducción y la creación van hilando el universo simbólico con el que el individuo y lo colectivo interactúan. Atada a esta tensión, se encuentra el liderazgo, pues yace inmerso entre las potencialidades, retos, dificultades, aciertos y aprendizajes con que se construye el ser humano cotidianamente.

La conformación y disputa por el orden social las realizan los sujetos sociales mediante la producción y transformación del mismo. Aunque, si bien la producción de lo social se realiza a través de un cierre hegemónico mediante una sutura y articulación -que posibilita la condición ontológica de la sociedad- éste se enfrenta a la imposibilidad de borrar por completo las huellas de la contingencia y debe lidiar con espacios de libertad, situación que termina por generar una dislocación en los sujetos que la producen (Fernández, 2012, p. 44).

El liderazgo emerge de los pliegues que se presentan en la tensión entre el sujeto y lo colectivo, lo que lleva a indagar por los mecanismos qué hacen posible dicha emergencia en los ámbitos de lo social y lo comunitario. El liderazgo no está determinado por las capacidades innatas de las personas, como se sostenía bajo la perspectiva de los grandes héroes, pero tampoco puede ser reducido a factores externos que sirven de detonantes o a un ambiente propicio para su realización: ambas posturas en extremo son nocivas. De ahí que el liderazgo deba ser entendido dentro de la tensión que demarca la sociología clásica francesa (Durkheim, 1976), a partir de vislumbrar la forma en que un individuo, como unidad biológica, se constituye en un ser social.

Desde esta postura, el liderazgo es asumido como una forma de relación social que se manifiesta a partir del desarrollo y uso de ciertas capacidades y habilidades para potenciar el trabajo adaptativo (Heifetz, 1993) logrando que las personas movilicen recursos, problematicen realidades, obtengan logros y desarrollen estrategias. La realidad es una construcción social (Berger \& Luckmann, 2001) y el liderazgo es una de las actividades con las que dicha construcción se lleva a cabo, pues teje vínculos intersubjetivos entre las personas y crea sentidos que re- significan el orden social.

Aun cuando los estudios sobre el liderazgo no poseen una delimitación que especifique conceptualmente al líder, se pueden describir algunos factores que permiten clasificar a los diversos tipos de líderes a partir de aspectos que van desde su personalidad hasta su capacidad para actuar frente a determinadas situaciones. Algunos de dichos factores se pueden reflejar en los siguientes enfoques:

- Enfoque de rasgos: se determina al líder dependiendo de ciertas características como lo son los altos niveles de energía, la tolerancia al estrés, la capacidad de decisión y la gestión del riesgo calculado. Como lo señala Lupano, "este enfoque permite determinar qué tipo de persona es la indicada para ocupar puestos de liderazgo, pero no indica si el líder será exitoso o no" (2003, p.109).

- Enfoque conductual: se basa en la percepción que tienen los seguidores de los líderes a través de dos categorías independientes. La primera, tiene que ver con la estructura, el definir roles, acomodar tareas y demás. La segunda, se relaciona con la percepción a través de la consideración que tienen los integrantes del grupo de la personalidad o forma de trabajo del líder hacia sus subordinados. $\mathrm{Su}$ fin es generar buenas relaciones entre el líder y el seguidor, e incluye valores como el respeto, la confianza, el compromiso, entre otros (Lupano, 2003, pág. 110).

- Enfoque situacional: depende mucho de los comportamientos de cada líder frente a diferentes situaciones que experimenta el grupo, debido a que no siempre se puede responder de la misma forma ante determinadas situaciones puesto que cada situación está supeditada a la indescifrable eventualidad de las contingencias (sin olvidar que cada persona tiene una visión del mundo irrepetible e indeterminada). 
- Enfoque transformacional: es uno de los más estudiados en los últimos años debido a su afinidad con la ética y la moral. A diferencia de los anteriores, su componente central no se encuentra en la figura del líder sino en el grupo y su capacidad para el cambio a la hora de afrontar los conflictos y problemáticas que se presentan. Los rasgos morales del líder potencian el cuestionamiento del accionar del grupo e invitan a una reflexión orientada a la transformación de la realidad inmediata con el ánimo de hacerla mejor.

Una lectura más profunda de los anteriores enfoques, a la luz de los aportes de Zalles (2010), da cuenta de dos grandes paradigmas que han abordado los estudios sobre el liderazgo. Por un lado, el tradicional, donde convergen los enfoques y corrientes de pensamiento que ven el liderazgo como un rasgo natural de algunos seres humanos ("los grandes hombres" que han guiado a la humanidad), o como producto de las relaciones que establecen las personas a partir del contexto o la situación que experimentan ("situacionalista") tal y como lo señala Yukl (2012). Y, por otro lado, el nuevo paradigma, centrado en la capacidad del líder de potenciar el trabajo adaptativo, el cual deviene en un conjunto de acciones que transforman e inspiran a los miembros del grupo a problematizar su realidad y restituir el equilibrio.

En este sentido, no se puede hablar de varios liderazgos; realmente existe uno solo que posee muchas formas de manifestación: tantas como interacciones humanas se presenten. Por eso el liderazgo social y el comunitario comparten un entramado complejo de significaciones y actividades que otorgan cargas apreciativas a uno y a otro. Estas, los identifican pero no los diferencian ni, mucho menos, los constituyen en unidades separadas. Es decir que las relaciones sociales que experimentan las personas, enmarcadas en la lógica del liderazgo, pueden llegar a convertirse en acciones que permiten caracterizar, por una parte, el liderazgo social del comunitario, sin que esto signifique su atomización y reducción y, por otra parte, comprender las mutaciones que el liderazgo presenta en las prácticas sociales que realizan las personas en diversos contextos y ámbitos de acción.

De ahí que el liderazgo social y el comunitario se constituyan en los saltos o mutaciones que presenta el liderazgo como actividad en el entramado social. No siendo estos más que los diversos pliegues en que el liderazgo se manifiesta en el complejo entramado de la realidad social.

En términos generales, para el proceso de clasificación, como fue mencionado anteriormente, se tuvieron en cuenta aspectos como: número de publicaciones, tipos de documentos, énfasis en el liderazgo social y el liderazgo comunitario, metodología utilizada y pronunciamiento en torno al sujeto como líder. Se evidencia que el grupo de investigación "Emprendimiento y Gestión de empresas de Familia” es el grupo que más ha producido documentos entre el 2004 y el 2014, con 24 productos realizados en total, en los que hay 18 artículos de revista y seis capítulos de libro. Este grupo está clasificado en la categoría $\mathrm{C}$ de Colciencias y tiene el mayor número de publicaciones realizadas entre los años 2010 y 2011. Así también, se encuentra vinculado a la Universidad de la Salle y es liderado por la docente Clemencia Navarrete Jiménez.

A este grupo de investigación le siguen, en cuanto a número de publicaciones, el grupo "Ciudadanía, paz y desarrollo", clasificado en Colciencias en la categoría $\mathrm{C}$, $\mathrm{y}$ perteneciente a la Corporación Universitaria Minuto de Dios, siendo su líder el docente Oscar Useche Aldana. Cuenta con un total de 18 productos, entre los que se encuentran ocho artículos de revistas, tres capítulos de libro, y siete libros. El año de mayor publicación de documentos de este grupo fue el año 2010, en el que realizaron diez publicaciones.

El tercer grupo de investigación con mayor número de publicaciones registradas es el grupo "Trabajo social, equidad y justicia social", clasificado en la categoría B de Colciencias, cuyo líder es la docente Rosa Ludy Arias Campos. Este semillero pertenece a la Universidad de la Salle y su corpus 
documental está conformado por 12 artículos de revistas y tres capítulos de libro, siendo el 2012 el año en el que más registra trabajos.

En contraste, durante este análisis se encontró que el "Grupo de Investigación en Desarrollo Social", clasificado en la categoría D de Colciencias y perteneciente a la Corporación Universitaria Iberoamericana, produjo solo un artículo de revista, realizado en el año 2008. . Así también, el grupo "Crecimiento Económico y Equidad con Responsabilidad", clasificado en la categoría de "reconocido" en Colciencias y vinculado a la Universidad San Buenaventura, registra un solo artículo relacionado con el objeto de estudio.

Al clasificar la información según el número de publicaciones realizadas por instituciones que avalan los grupos de investigación, se encontró que en primer lugar (en orden descendiente) se encuentra la Corporación Universitaria Minuto de Dios, que sobresale con 41 publicaciones entre las que hay 21 artículos de revistas, ocho capítulos de libro y 12 libros. Los grupos responsables de dicha producción académica fueron: el grupo "Ciudadanía, Paz y Desarrollo", que como se mencionó anteriormente realizó 18 publicaciones; el grupo "Construcción de Ciudadanía, Comunidad y Tejido Social - CRISÁLIDA", que produjo diez publicaciones; el grupo "Innovaciones Educativas y Cambio Social", que participó con nueve publicaciones $\mathrm{y}$, por último, el "Grupo de Investigación en Lenguaje, Comunicación y Participación", con cuatro publicaciones. Así, es importante reconocer el papel protagónico que desarrolla esta universidad en estudios sobre el liderazgo social y comunitario.

La Universidad de la Salle también ejerce un rol protagónico, en cuanto a la cantidad de publicaciones, con 39 documentos en su haber, realizadas por el grupo "Emprendimiento y Gestión de empresas de Familia", que realizó 24 publicaciones, y el grupo "Trabajo social, equidad y justicia social”, que realizó quince.

Dirigiendo la mirada hacia la producción bibliográfica por años, se destaca que el 2010 fue el año con mayor producción de documentos con 35 publicaciones en total, entre las cuales se encuentran 24 artículos de revista, ocho capítulos de libros y tres libros. Para el año 2008, el rastreo puso en evidencia la producción de 27 publicaciones relacionadas con el interés investigativo del semillero, ubicando a este año como el segundo en el que más se trabajó sobre liderazgo social y comunitario; en este caso se registran 27 publicaciones distribuidas en trece artículos de revista, cuatro capítulos de libro y diez libros. En tercer lugar, se ubica el año 2012, que registra 18 artículos de revista, cinco capítulos de libro, y tres libros.

Este rastreo mostró que para el año 2004 la producción académica en el tema es considerablemente baja, pues solamente se registraron tres artículos de revista, un capítulo de libro y dos libros.

Revisando de forma general la información anterior, se evidencia que la producción de artículos referentes al liderazgo fue muy baja en el 2004; ello lleva a preguntarse: ¿era este un tema de poca trascendencia para la época? Así, en contraposición a la baja producción académica de este año, se encuentra un aumento del número de publicaciones entre los años 2006 y 2008 (como es posible evidenciar en la cantidad de libros producidos en el año 2008) Sin embargo, en el año 2009 se registra un descenso en la producción académica por cada grupo, no obstante, es el año 2010 el que llama la atención, pues los datos encontrados conducen al siguiente interrogante cuando se analizan los textos de estudio: ¿Qué elementos pudieron influir en el incremento de investigaciones sobre el liderazgo social y comunitario luego del año 2010 ? Interrogante que ya había sido planteado en la primera trayectoria relacionada con la identificación y selección del corpus documental.

Una de las hipótesis frente al interrogante anterior, parte de reconocer la normatividad sobre responsabilidad social que rige al país desde la década pasada. A mediados del año 2000, las Naciones Unidas, en cooperación con grandes multinacionales, tomaron la decisión de dar lugar 
a un movimiento que aumentara la responsabilidad social en el planeta: el Pacto Mundial, un acuerdo que buscaba fomentar la realización de nuevas obras sociales de todas las empresas en pro del beneficio de las poblaciones desfavorecidas del mundo (ONU, 2000).

Teniendo en cuenta esto, dicha normatividad empezó a tener importancia en Colombia a mediados del año 2010, época en la cual el Gobierno impulsó a las empresas a fomentar programas de responsabilidad social empresarial con el fin de implementar lo pactado en el Pacto Mundial a principios de siglo. Se evidencia que este pacto, además de impulsar la responsabilidad social, incentivó la realización de más investigaciones de tipo documental sobre responsabilidad social empresarial, lo que influyó en el aumento del número de publicaciones en los años 2010, 2011 y 2012.

Dentro de este análisis descriptivo, también se tuvieron en cuenta los autores con mayor número de publicaciones y sus vínculos con grupos de investigación e instituciones que avalan dichos grupos. Al revisar el número de producciones por año, el rastreo permitió hacer una caracterización por autores. En este sentido, se encontró que la investigadora Yolanda García, quien hace parte del grupo de investigación "liderazgo" (clasificado en la categoría $\mathrm{C}$ en Colciencias) y se encuentra vinculada a la Universidad Militar Nueva Granada, se presenta como la mayor productora de publicaciones con once publicaciones, cuatro libros y siete artículos de revista. . En segundo lugar, se encontró al investigador Jorge Alberto Gámez Gutiérrez, del grupo de investigación "Emprendimiento y gestión de empresas de familia", clasificado en la categoría $\mathrm{C}$, y perteneciente a la Universidad de la Salle, quien ha publicado ocho artículos de revista y un capítulo de libro (es decir, un total de nueve publicaciones). En tercer lugar, se ubicó al docente investigador Oscar Useche Aldana, vinculado al grupo de investigación "Ciudadanía, paz y desarrollo”, perteneciente a la Corporación Universitaria Minuto de Dios; este grupo de investigación desarrolló 3 artículos de revista, un capítulo de libro y tres libros, para un total de siete publicaciones en las que el profesor Useche aparece como auto o coautor.

Teniendo en cuenta la información mencionada anteriormente, se destaca el predominio de las universidades de La Salle y la Corporación Universitaria Minuto de Dios en cuanto a temas de liderazgo social y liderazgo comunitario. Particularmente en sus programas de trabajo social y administración de empresas, y en las iniciativas alrededor de la educación social para la paz, la ciudadanía y el desarrollo social.

Los estados del arte se caracterizan porque, lejos de ser una mera aglomeración de datos, intentan dar cuenta de los sentidos teóricos que estos datos potencian (Arellano, 2012). Así, las reflexiones presentadas a continuación se constituyen en una reflexión que apunta a la construcción de los sentidos teóricos que identifican y caracterizan el corpus documental. La mirada general a los elementos teóricos que identifican a los grupos de investigación referenciados permite discernir que existe una fuerte orientación hacia el liderazgo centrado en el trabajo adaptativo. Es importante señalar que si bien no son explicitas las referencias a autores como Antonakis (2012), Burns (1978), Heifetz (1993), Bass \& Avolio (2004), sus postulados se pueden identificar de forma reiterada. El liderazgo inspiracional y el liderazgo transformacional son elementos comunes (Vásquez, 2013), por lo que en la gran mayoría de documentos los autores reconocen la importancia de motivar el trabajo en los miembros de las organizaciones que las componen; sobre todo, aquel en el que estos se ven obligados a generar estrategias y acciones que posibiliten solucionar las dificultades a las que se enfrenten y satisfacer sus necesidades.

El liderazgo debe permitir que las personas sean quienes reconozcan sus propios problemas $\mathrm{y}$ agencien las respectivas soluciones. Los rasgos de la personalidad y el manejo de las emociones son aspectos que complementan el carácter del sujeto líder. Ya sea a través del componente ambiental, educativo o empresarial, el liderazgo 
es concebido como una estrategia para impulsar la acción colectiva. Esta postura deja por fuera las visiones tradicionales que reducían al liderazgo a un ejercicio de autoridad y poder basado en el culto a la persona y en la participación limitada.

De la misma forma, un elemento teórico común que se encontró en los documentos registrados es del líder como pedagogo, dado a que el liderazgo requiere ciertas capacidades pedagógicas sobre todo en el trabajo comunitario y empresarial: la socialización de los resultados y la construcción de iniciativas requieren el manejo de grupos. En este sentido, los trabajos de Yolanda Guerra sobre el liderazgo eficaz sugieren aspectos pedagógicos indispensables en tales propósitos. Igualmente, los estudios presentados por los grupos "Trabajo social, Equidad" y "Justicia Social", de la universidad de la Salle, y "Construcción de Ciudadanía, Comunidad y Tejido Social" de la Corporación Universitaria Minuto de Dios, comparten en sus pesquisas el reconocimiento de lo pedagógico tanto como un factor preponderante en la formación de nuevos líderes, como estrategia para el trabajo comunitario en organizaciones (Juliao, 2013).

La responsabilidad social es otro de los componentes que identifican las apuestas teóricas de los grupos indagados desde los abordajes conceptuales en los que se discuten perspectivas teóricas e históricas como se ve en Uribe (2005) y en Estrada (2007), hasta elementos que conforman una deontología de la responsabilidad social, como lo muestran Pérez (1998) y Garavito (2005). Los estudios revisados abordan, desde el ámbito empresarial e institucional (universidades), la responsabilidad como un compromiso que asumen las organizaciones con las comunidades y las poblaciones donde su trabajo tiene impacto.

Después de organizar y analizar el corpus documental, se procede a la construcción del sentido global teórico, del que emergieron cuatro horizontes que despliegan las formas de comprender el liderazgo desde unas posturas cercanas a la filosofía política y a la sociología.
El primero de ellos pertenece al plano del poder, entendido como la fuerza capaz de orientar la voluntad de los individuos. Ya sea a través del mandato, la subordinación o la cooperación, el poder está implícito en las personas: es la piel que cubre las interacciones humanas y, por ende se encuentra en todas partes (Foucault, 1988). Lo cual significa que el poder hace posible la emergencia del liderazgo, ya que éste atraviesa todo el mapa de las relaciones humanas, de ahí que la figura del líder no pueda ser comprendida sin la relación de fuerzas amparadas en el reconocimiento de los individuos como miembros de un grupo. Tradicionalmente el liderazgo se relaciona con la imagen de la autoridad, sobreentendiéndose que todo líder la posee dado que esta es requerida para ejercer su poder (Rojas, 2005). Sin embargo, los aportes dados por Heifetz (1993) y por la Escuela del enfoque transformacional sostienen que puede ejercerse el liderazgo sin autoridad, lo que conlleva a una ruptura con el modelo tradicional. El liderazgo sin autoridad posibilita que las personas puedan asumirse como sujetos libres, estableciendo relaciones de cooperación en las que el trabajo adaptativo, orientado a la satisfacción de necesidades o al abordaje de problemas, sea considerado como una herramienta efectiva para las organizaciones. Esto significa que, independientemente del ejercicio de la autoridad, el liderazgo se enmarca en las dinámicas y posibilidades del poder como relación.

El liderazgo como relación social se encuentra sometido a dinámicas y tipos de poder que hacen parte del universo simbólico de lo social. El liderazgo se configura como una de las formas en que el poder se manifiesta en la realidad social, pero escapa a los vínculos tradicionales de dominación y subordinación que diluyen la figura del líder para poner en escena la del tirano o dictador, así como la del grupo que, sumiso y subyugado, obedece sus dictámenes. Para Heifetz (citado por Moreno):

(...) el liderazgo se asocia con una actividad que algunas personas realizan, con el fin de movilizar a otras personas, mientras que la autoridad, 
tanto formal como informal, es un status concedido por las personas, quienes se sustentan en la idea de que la autoridad debe hacer lo que ellos esperan. No obstante, en el liderazgo adaptativo no se trata de cumplir o de superar las expectativas de los autorizadores; se trata de cuestionar algunas de esas expectativas y de buscar una forma de decepcionar a las personas, situándolos en la realidad (Moreno, 2014, p. 15).

El segundo horizonte se refiere a la capacidad que poseen las personas, en sus interacciones con otros sujetos, de orientar su conducta hacia el logro, la motivación y la gestión de un conjunto de propósitos. El poder nuevamente se encuentra presente, pero en este caso la pregunta que orienta la discusión gira en torno a qué elementos determinan o influyen para que al interior de un grupo que ha vivenciado las mismas experiencias y recorrido las mismas tramas de la vida, surja una persona que asuma la voz del grupo, ya sea por el reconocimiento que este le otorga o porque logra movilizar con su carisma al punto de entusiasmar y alentar las acciones del mismo:

(...) el líder debe poner el ejemplo haciendo las cosas, cuando esto sea posible y su habilidad, destreza o facultad así se lo permitan. Lo anterior hace que las personas ganen confianza en él. Con su actuar da garantía y estimula a los demás a emularlo, evita los pretextos e invita a refrendar las acciones de manera colaborativa (Barber \& Clúa de la Torre, 2014, p. 8).

Para presentarlo de forma más clara, veamos la siguiente vivencia, tomada de una de las experiencias registradas en la revisión documental: Los habitantes del sector de Brasilia, en el sur de la ciudad, vivían cerca al antiguo relleno sanitario de Gibraltar. Al verse enfrentados por este hecho a una emergencia ambiental, se organizaron para adelantar acciones de resistencia y movilización que permitieran su cierre. Todos los habitantes compartían historias comunes como el desplazamiento, la informalidad laboral, dificultades de accesos a servicios de salud y educación, entre otros (Torres, 2011). Sin embargo, ante un acontecimiento que afecta a la comunidad emergen líderes comunitarios que direccionan colectivamente al barrio: ¿Qué lleva a que algunos sujetos hayan asumido tal rol si todos compartían las mismas historias comunes y experimentaron la situación que desencadenó la movilización?

La emergencia del liderazgo no es arbitraria, pero tampoco debe ser reducida a la lógica instrumental que explica los fenómenos sociales por sus causas y efectos. El liderazgo es un pliegue que emerge de la tensión entre la subjetividad y la colectividad, las cuales encuentran en los acontecimientos los momentos para su despliegue. Cada persona re-significa las prácticas sociales que vivencia $\mathrm{y}$, en este proceso, incorpora o elimina marcas que lo identifican y que, por ende, anudan su personalidad. Esto significa que no se nace líder, ni mucho menos con habilidades para el liderazgo. Por el contrario, es el conglomerado social donde las intersubjetividades encuentran la posibilidad de tejer redes de relación en las que se desarrollan las capacidades para ejercer el liderazgo. De ahí que el estudio del liderazgo exija comprender este fenómeno como una construcción sociocultural que oscila entre las dimensiones subjetivas y objetivas en que se presenta en la compleja red del entramado social (Collado et al, 2016).

La capacidad para posibilitar que las personas al interior de un grupo logren problematizar su realidad inmediata y atiendan acertadamente sus necesidades, de manera que logren satisfacerlas, se constituye en la principal meta del liderazgo (Heifetz, 1993). Para lograr esto, no se requiere de una posición de poder que direccione la conducta de las personas hacia tales logros, como tradicionalmente lo hace una autoridad, sino que se debe disponer de las habilidades discursivas y prácticas que permitan motivar cambios en las conductas del grupo.

Cada persona, desde su rol y posición en el grupo, puede constituirse en el vértice que movilice, a través de su ejemplo o direccionamiento discursivo, la participación de las demás personas para generar iniciativas de cambio en la realidad inmediata. Este esquema de trabajo ha sido muy 
aceptado en las organizaciones sociales en las que las personas tienen voz y decisión en las iniciativas que se proponen y validan:

[...] toman especial significado los rasgos personales, entre otros casi siempre se habla de honestidad, integridad, confianza, buenos hábitos, razonamiento analítico. Estas características son indiscutiblemente importantes, pues con ellas el líder puede actuar en los siguientes pasos, como la movilización de la dedicación individual (es decir, hacer que otros se comprometan) y su capacidad para engendrar a la organización (lo que significa su habilidad para poder formar verdaderos equipos que administren el cambio) (Barber \& Clúa de la Torre, 2014, p.47).

El tercer y el cuarto horizonte que permite comprender el liderazgo, en sus acepciones sociales y comunitarias, lo componen, por una parte, las relaciones sociales en las que la interacción permite la formación de unos pliegues intersubjetivos en los grupos humanos $\mathrm{y}$, por otra parte, los pliegues subjetivos, en los que intervienen los aspectos cognitivos, emotivos y valorativos de las personas al relacionarse con sus semejantes en los vínculos asociativos y colectivos.

Para el horizonte en el que se visibilizan los pliegues intersubjetivos, las organizaciones $\mathrm{y}$ comunidades funcionan como estructuras que inciden en el comportamiento de las personas. Cada agrupación funciona como una micro-sociedad en la que se reproducen los aspectos ideacionales y los sistemas de creencias. De ahí que se presenten comportamientos y conductas que visibilizan la esfera social: "La sociología de la organización ha delimitado su problema a partir del reconocimiento de que las organizaciones constituyen un sistema social" (Gonnet, 2014, p. 230-231).

Bajo esta perspectiva, cada organización posee unas normas o reglas explicitas o implícitas que regulan el microsistema social y posibilitan el equilibrio del grupo. Cuando se presentan fracturas en el sistema de reglas, como por ejemplo una alteración o falta a la norma, se crea un desequilibrio que afecta a todo el grupo.
Con el ejercicio del liderazgo, usualmente las reglas se refuerzan o conforman, pero es muy poco probable que se altere todo el microsistema normativo dado que esto daría lugar a una escisión o resistencia en la agrupación. Al respecto, es importante señalar que

(...) las organizaciones son sistemas sociales autopoiéticos, en el seno de los cuales las únicas operaciones posibles son las decisiones. A pesar de que estas son secuencias de acontecimientos que permiten la siguiente decisión, no puede hablarse de una finalidad de la organización a la que estarían subordinadas. Como sistema autopiético, una organización tiene fronteras y elementos que no son datos objetivos sino productos propios de la organización (Urteaga, 2009, p. 306).

El liderazgo y el rol del líder juegan un papel importante en los momentos de autopoiesis de las organizaciones, pues inciden el caleidoscopio del grupo y buscan mantener el equilibrio del mismo. Cuando el líder logra comprender el sistema normativo y de valores que posee el grupo, amplía la posibilidad de direccionamiento y acatamiento de su gestión, ya que las probabilidades de aceptación a sus lineamientos se encuentran matizadas por la legitimidad de su rol y su posición en la organización. Cada líder es influenciado por el colectivo; de hecho, el ejercicio del liderazgo como proceso se encuentra matizado por las tensiones, avenencias y resistencias que se presentan entre el individuo y la colectividad.

Finalmente, el pliegue subjetivo hace referencia al desarrollo de habilidades y capacidades que posee la persona que ejerce el liderazgo. Estas conllevan a reconocer al ser humano como un ser de acción que se moviliza a partir de lo que conoce (lo cognitivo), lo que siente (lo emotivo), y el sistema de valores que orienta sus creencias (lo valorativo). Estas tres perspectivas se constituyen en los aspectos a tener en cuenta cuando las personas gestionan acciones en una organización.

Cada persona deja una huella o marca sobre el grupo, así como el grupo influye en él. Estas marcas están determinadas no solo por los conocimientos 
involucrados en el ejercicio de su gestión, sino también por los sentimientos y las cargas valorativas que la persona asigna a sus prácticas sociales. Así lo expresa la profesora Natalia Canto:

Cuando se inicia el camino del liderazgo se inicia de forma bastante ciega y confusa, y es después, con el paso de los años y la reflexión, que los mismos protagonistas empiezan a ver gestos de liderazgo en su papel de delegados de curso, en su papel de activismo y de organización de huelgas y revueltas en el instituto, en sus primeras actividades como voluntarios (Canto, et al., 2009, p. 55).

Cada persona logra ejerce influencia en un grupo a partir de los aspectos descritos anteriormente. Si posee amplios conocimientos para planear y ejecutar proyectos, se le facilitará su desempeño en el grupo; sin embargo, debe involucrar un equilibrio en sus emociones que le permitan el direccionamiento sobre los miembros del mismo. Si sus sentimientos o su sistema valorativo choca con los sentimientos y creencias de las personas, su direccionamiento se verá truncado. Es decir que de nada le servirá a la persona el conocimiento si no posee la facultad para comunicarlo y motivar a los demás a realizar lo que se desee o necesite. El carácter y la personalidad marcan determinadas pautas para el desarrollo del liderazgo, presentándose estos entre las tensiones que se dan entre el sujeto y los integrantes del grupo. En este sentido, cada persona le imprime un rasgo particular al liderazgo como proceso, a partir de los rasgos subjetivos que se desenvuelven en el marco de las relaciones intersubjetivas.

Como ha sido mencionado anteriormente, el liderazgo social y el liderazgo comunitario son, cada uno, una cara de la misma moneda. Las orientaciones y las formas como desempeñan sus roles las personas al interior de los grupos, otorgan marcas y características particulares a las maneras en las que se manifiestan el liderazgo social y el liderazgo comunitario. De ahí que tanto en uno como en otro predomine una racionalidad que se caracteriza por la lógica de la estrategia, el planeamiento y el riesgo calculado, cercana a los esquemas del ámbito empresarial y académico, y asumida desde el liderazgo social como responsabilidad. Los seres humanos establecen relaciones grupales en las que definen roles a partir de su desempeño y posición en el grupo. Entre más especializado sea su papel, tantas más estructuras jerárquicas matizarán las dinámicas del grupo, dando forma a fuertes divisiones sociales al interior del mismo. Max Weber denominaría bajo esta perspectiva el trabajo burocrático, el cual se caracteriza por ejercer relaciones de liderazgo típicamente societarios. El liderazgo social estaría relacionado con la racionalidad de fines y de valores de Weber; en tal sentido, la compensación, el rol y la posición se convierten en elementos de suma importancia a la hora de comprenderlo. Así también, agrega un nuevo sentido que lo distingue, tal y como menciona la profesora Mila Natalia Canto, refiriéndose al líder social:

(...) todo aquel o aquella que trabaja (de forma remunerada o voluntaria) en áreas diversas de nuestra sociedad; actuando como catalizador del cambio social; realizando, inspirando o ideando proyectos compartidos en el seno de una entidad u organización; proyectos a menudo innovadores o desarrollados de forma innovadora, que se dirigen al bien común (Canto, et al., 2009, p. 13).

Por su parte, en el ámbito comunitario la racionalidad se destaca por ser un poco más pragmática y estar orientada a la atención de necesidades colectivas enmarcadas en la lógica de la contingencia y el abordaje de las problemáticas sentidas. Las luchas sociales por el derecho a la ciudad de los años 60 y 70 son un buen ejemplo de la conformación de un liderazgo comunitario. Los campesinos desplazados por la violencia política y los procesos de modernización, bajo los estandartes del desarrollo y el progreso de la nación, llegaron a unas ciudades que no tenían la infraestructura habitacional ni de servicios para recibirlos. De ahí que encontraran en las periferias lugares para asentarse y emprender una nueva forma de vida. Sus historias estaban escritas por la misma pluma, pues eran hijos y nietos de la violencia de los años 40 y 50, en medio de la que personas cansadas de la exclusión política buscaban un pedazo de tierra donde tender sus sueños. Fue 
así como se dio una urbanización extendida y exponencial de las ciudades que, al carecer de todo tipo de control, pronto se vieron "invadidas" por nuevos pobladores que fueron vistos como un peligro para la sociedad. La apropiación colectiva de lotes, redes de energía eléctrica y la construcción de aljibes para el suministro de agua fueron acciones defensivas con las que se movilizaron colectivamente hacia su derecho a la ciudad. Las luchas por el transporte público, la legalización de sus barrios, la construcción de centros educativos y puestos de salud, y demás, se anudan a las acciones que sirvieron la emergencia de un liderazgo comunitario que, sin embargo, no ha estado ajeno a las formas tradicionales de poder como el clientelismo y la cleptocracia.

El papel del liderazgo ha sido importante en la historia del trabajo comunitario porque desde él fue posible levantar gran parte de los asentamientos subnormales y populares de la ciudad a través de las invasiones, a veces la única vía para acceder a lotes donde levantar los ranchos; gestionó recursos para mejorar las condiciones de las comunidades y abrió espacios a la actividad política y participación de la mujer en estas actividades (Negrete, 2008, p. 14).

Igualmente, se presenta un componente axiológico cercano a las manifestaciones de racionalidad mencionadas anteriormente. En el liderazgo social predominan valores donde se refuerzan las acciones individuales de las personas como la puntualidad, la honestidad, la disposición para el trabajo en equipo, el cumplimiento de las responsabilidades, etc. Mientras que en el liderazgo comunitario los valores se orientan hacia el estímulo de las acciones colectivas, resaltando los aprendizajes de los procesos vividos como la cooperación, la solidaridad y la amistad.

\section{Pliegues discursivos: irrupciones filantrópicas y compensaciones colectivas}

La filantropía, en su acepción más general, se entiende como aquella acción desinteresada que desarrollan algunos seres humanos en favor de otros. Sin embargo, es posible evidenciar que sus propósitos pueden variar dependiendo de los actores y sus acciones. En este sentido, en el ámbito del liderazgo social, la filantropía adopta una forma de servicio con la que busca afrontar las consecuencias de un problema sin ir a su raíz, surtiendo así una surte de asistencialismo. Por su parte, en la esfera del liderazgo comunitario, la filantropía se configura a partir de trabajos y esfuerzos que guardan mayor cercanía con la búsqueda de soluciones a los problemas desde sus causas. Para ilustrar lo anterior, piénsese en un problema particular como el hambre: mientras que para algunos actores este se soluciona mediante la entrega de alimentos a aquellos que los necesitan, para otros las acciones se enfocan en procesos de organización que buscan garantizar el derecho a la alimentación de todos.

Lo anterior permite aducir que, si bien el liderazgo social y el liderazgo comunitario, como ya se ha mencionado, no son dos procesos inversos, sí es posible evidenciar pliegues discursivos en torno al enfoque y al alcance de sus acciones.

Finalmente, cabe mencionar que, durante el recorrido hecho, ha sido posible encontrar que las investigaciones de tipo documental que guardan relación con las discusiones teóricas son las de mayor predominancia en las investigaciones que abordan el liderazgo social. A su vez, las investigaciones cualitativas donde se destaca el trabajo de campo, las entrevistas a profundidad y la sistematización de experiencias se constituyen en los elementos predominantes en el liderazgo comunitario.

El sujeto como "líder" emerge bajo la figura del experto o profesional que asume su rol a partir del reconocimiento de su idoneidad en el campo de trabajo. Se le considera como la persona capaz de orientar y motivar a otros para alcanzar metas en las que el fin último es el mayor beneficio colectivo. Las empresas o universidades que desarrollan procesos de responsabilidad social, contratan en muchos casos a profesionales "lideres" para que intervengan en un determinado tipo de población 
y asuman acciones de liderazgo. Estos adoptan la figura de "líder social" y desarrollan estrategias de acción centradas en una racionalidad técnica y especializada marcada en la planeación, ejecución y evaluación de programa o procesos. Su nivel de acierto esta medido por la capacidad que posean para lograr que el grupo los sigan y apoyen. Así lo señala la profesora Iliana Páez y su grupo de investigación al referirse a las competencias del dirigente líder:

Las organizaciones requieren de líderes, personas que sepan orientar y dirigir, con responsabilidad, los esfuerzos de sus colaboradores para cumplir con eficacia los objetivos -y las metas comerciales, financieras productivas generadoras de valor-en aras de garantizar su crecimiento $\mathrm{y}$ asegurar su futuro. Las organizaciones tienen hoy en día una gran responsabilidad frente a lo social y ambiental debido a que la sociedad es más exigente, y espera permanentemente resultados innovadores de sus líderes organizacionales, a quienes están juzgando constantemente (Páez, González, Enciso, Perilla \& Martínez, 2012, p. 28).

Por otra parte, en el liderazgo comunitario el semblante del sujeto líder se encuentra matizado por el contexto, el cual le brinda las pautas de orientación para su trabajo inundando de cierto pragmatismo su accionar. El territorio, como la manifestación de la realidad a transformar, lleva a una re-significación de los procesos sociales y a la construcción de nuevos vínculos identitarios que potencian el empoderamiento colectivo; así lo señala Torres:

(...) el líder de los grandes movimientos populares es aquel que posee una sensibilidad plástica de captar y resumir, en un momento dado, el impulso que labora el agitado subfondo del alma colectiva; aquel que se convierte en antena a donde ascienden a buscar expresión, para luego volver, metodizados al seno de donde ha salido, las demandas de la moral, lo justo, de lo bello, en el legítimo empeño humano de avanzar hacia mejores destinos (Torres \& Barragan, 2012, p. 17).

\section{Conclusiones}

Aun cuando se han producido documentos e indagado en ellos en relación al liderazgo desde distintos campos y enfoques, no existe un ejercicio reflexivo en torno a la percepción, inclinación y los rasgos característicos que componen tanto el liderazgo social como el liderazgo comunitario. En esta medida, preguntarse por el estado de la cuestión, usando como metodología el estado del arte, se convierte en la posibilidad de reflexionar sobre los usos, impactos y trascendencias que tiene, para el mundo social y científico, la forma en la que se comprende y se pone en práctica el liderazgo. Para el ámbito educativo que nos ocupa como universidad, este proceso investigativo da luces sobre los posibles cambios en la forma de entender el liderazgo, así como sobre los propósitos y alcances que tiene el hecho que desde la academia se esté aportando a la configuración de un tipo de liderazgo.

Durante el desarrollo de la investigación fue posible evidenciar que, si bien existe amplia documentación escrita sobre el liderazgo, no se encuentran documentos que den cuenta de la relación de esta categoría con lo social y/o lo comunitario específicamente, pues muchos de los trabajos centran su mirada en los procesos de formación del líder sin señalar ninguna distinción particular. En este sentido, el liderazgo es tomado indistintamente, lo que denota poca o ninguna preocupación por diferenciarlo.

Tanto el liderazgo social como el liderazgo comunitario, están definidos por su orientación al desarrollo personal y colectivo de los integrantes del grupo, por lo que sus propósitos pueden variar no solo dependiendo de las características de cada uno de los sujetos que fungen como líderes, sino también por las características de quienes legitiman el liderazgo.

La dinámica global que buscaba impulsar nuevas formas de responsabilidad social, desde el ámbito empresarial y medio ambiental, tuvo 
cierta incidencia en la producción académica relacionada con el tema del liderazgo en el contexto colombiano.

En las investigaciones consultadas, predomina el liderazgo transformacional; sin embargo, el mismo se ajusta a las necesidades del enfoque social o comunitario que se estén abordando. Existen trabajos que hacen explicita esta característica, pero la gran mayoría no lo enuncia o no lo evidencia.

La concepción del sujeto como "líder" tiene variaciones según el enfoque que tenga la investigación; no obstante, es posible identificar elementos constantes tanto en el líder social como el líder comunitario. Así, mientras que el líder social guarda cercanía con la idea de un sujeto experto o profesional que debe estar en constante formación, el líder comunitario se asocia más con un sujeto formado desde la acción práctica donde el quehacer determina su capacidad.

Dependiendo del enfoque con el que se quiera abordar el liderazgo, existe una inclinación mayor o menor a utilizar determinadas metodologías. Así, al indagar en torno al liderazgo social predominan aquellas metodologías de tipo documental y teórico, mientras que al indagar sobre el liderazgo comunitario, la inclinación metodológica es de tipo cualitativo, donde se vincula el trabajo de campo y la sistematización de experiencias, entre otros.

\section{Referencias bibliográficas}

Antonakis, J. (2012). Transformational and Charismatic Leadership. Suiza: University of Lausanne.

Arellano, S.J. (2012). La originalidad de la Investigación en torno al Estado del Arte. Memorias del III Encuentro Latinoamericano de Metodología de las Ciencias Sociales. "Métodos alternativos o críticos de la investigación en ciencias sociales". Construcción de opciones metodológicas para las ciencias sociales contemporáneas. Universidad de Caldas, Manizales, Colombia.
Barber, K.C. \& Clúa de la Torre. C. (2014). Liderazgo Inspiracional. México: Mc Graw Hill.

Bass, B. \& Avolio, B. (2004). Multifactor leadership Questionnaire. USA: Mind Garden Inc.

Berger, P. \& Luckmann, T. (2001). La construcción social de la realidad. Buenos Aires: Amorrortu.

Burns, M.J. (1978). Leadership. New York: Harper y Row.

Canto, N., Castiñeira, A. \& Font, A. (2009). Las Fuentes del Liderazgo Social. Barcelona: Mediterraneo. S. L.

Collado, C.F., Jiménez, D.J. \& Entrena-Durá, F. (2016). El liderazgo político en las democracias representativas: propuesta de análisis desde el constructivismo estructuralista. Revista Mexicana de Ciencias Políticas y Sociales, 61 (228), 57-90.

Coser, L. (1961). Las Funciones del Conflicto Social. México. D.F.: Fondo de Cultura Económica.

Delgado, S. (2004) Sobre el concepto y el estudio del liderazgo político. Una propuesta de síntesis. Psicología Política, 29, 7-30.

Durkheim, E. (1976). Educación como socialización. Salamanca: Sígueme.

Estrada, M.S. (2007). Liderazgo a través de la historia. Revista Scientia et Technica, 13(34), 343-348.

Fernández, F.E. (2012) El Sujeto Politizado en la Segunda Mitad Del Siglo XX en Colombia (19561978). (Tesis de maestría). Universidad Distrital Francisco José de Caldas, Bogotá, Colombia.

Foucault, M. (1988). El Sujeto y el Poder. Revista Mexicana de Sociología, 50 (3), 3- 20.

Garavito, V.D. (2005). La Responsabilidad Social: una Ética de lo Público. Revista de Comunicación Educativa, 42, 20 - 27.

Gonnet, J.P. (2014). Cosmologías y orden social organizacional. Revista Sociológica, 29 (81), 227- 260. 
González, M.D. (2014). Los Estudios Organizacionales. Un campo de conocimiento comprensivo para el estudio de las organizaciones. Innovar Revista de Ciencias Administrativas y Sociales, 24 (54), 43-58.

Heifetz, R. (1993). Liderazgo sin respuestas fáciles. Barcelona: Paidós.

Juliao, V.C. (2013). Una Pedagogía Praxeológica. Bogotá: Corporación Universitaria Minuto de Dios.

Lupano, P.M. (2003). Estudios sobre el liderazgo. Teorías y evaluación. Revista Psicodebate, 6, (1), 107- 121.

Molina, I.J. \& Ramírez, R. (2009). Re-significando el territorio: el caso de la cuenca del Río Tunjuelo. Revista Mediaciones, 9 (1), 123 - 142.

Moreno, S.M. (2014). Demarcación del Concepto de Liderazgo Adaptativo, propuesto por el profesor Ronald Heifetz. (Tesis de pregrado). Universidad del Rosario, Bogotá D.C., Colombia.

Muñoz, M.G. (Sin fecha). Liderazgo. Centro de Humanidades - Formación Avanzada. Universidad Pontificia Bolivariana, Working Paper $\mathrm{N}^{\circ} 1$. Recuperado de: http://www.academia. edu/11575807/LIDERAZGO_El_liderazgo_es_ como_la_belleza_Dif\%C3\%ADcil_de_definir_ pero_f\%C3\%A1cil_de_Reconocer_si_uno_lo_ ve_W._Bennis_EL_LIDERAZGO.

Negrete, B.V. (2008). Liderazgo y Organización Comunitaria: una vía a la democracia y al desarrollo. Revista Documentos para la Reflexión, 3 (3), 47- 55.

ONU. (2000). Pacto Mundial. Organización de las Naciones Unidas. Recuperado de: http://www.pactomundial.org/.

Pacsi C.A; Estrada M. W; Pérez V. A. Cruz M. P. (2014). Liderazgo laissez faire. Revista de Investigación de Administración, 1 (1), 67-72.

Páez, G. I., González, A., Enciso, E. Perilla, L. \& Martínez, M. (2012). Diez competencias del dirigente líder. Un marco conceptual del enfoque del liderazgo transformacional. Bogotá: Universidad Externado de Colombia.

Palomo, V. M. (2010). Liderazgo y Motivación de trabajos en equipo. Madrid: ESIC Editorial.

Pérez, L.J. (1998). Liderazgo y ética en la dirección de empresas. Bilbao: Deusto.

Robinson, S. \& Kerr, R. (2009). "The symbolic violence of leadership: A critical hermeneutic study of leadership and succession in a Brithish organization in the post-Soviet context". Human Relations, 62 (6), 875-903.

Rojas, T.G. (2005). Psicología social y nuevo líder. Bogotá: Magisterio.

Sánchez, A. (2002). Dispositivos de empoderamiento para el desarrollo psicosocial. Universitas Pshycologica, 1 (2), 39-48.

Torres, C.A. (2011). Sentidos y prácticas de participación local desde los actores sociales. Revista Aquelarre, 10 (20), 175- 201.

Torres, C.A. \& Barragan, C.D. (2012). Liderazgo comunal como ciudadanía crítica. Programa formación de formadores para la organización comunal. Bogotá: Universidad Pedagógica Nacional y Presidenia de la República.

Uribe, B.Á. (2005). Ética, Responsabilidad social y Empresa. Bogotá: Escuela de ciencias humanas. Universidad del Rosario.

Urteaga, E. (2009). La teoría de sistemas de Niklas Luhmann. Contrastes Revista Internacional de Filosofía, 15, 301-317.

Vásquez, A. (2013). Interdependencia entre el liderazgo transformacional, Cultura organizacional y cambio educativo: una reflexión. Revista Iberoamericana sobre Calidad, Eficacia y Cambio en Educación, 11 (1), 73- 91.

Vélez, R.O. (2002). Investigación Cualitativa. Estado del Arte. Medellín: Universidad de Antioquia. Facultad de Ciencias sociales y humanas. 
Weber, M. (2003). Economía y Sociedad. México D.F.: Fondo de Cultura Económica.

Yukl, G. (2012) Leadership in Organizations. New Jersey: Prentice Hall.
Zalles, J.H. (2010). Liderazgo. Un concepto en evolución. Quito, Ecuador: Universidad San Francisco de Quito. 